\title{
The revolving door syndrome in internal medicine: a study on 11,846 subjects discharged from all Internal Medicine Departments of Tuscany with diagnosis of heart failure and pneumonia
}

\author{
Matteo Tellini, ${ }^{1}$ Alessandra Petrioli, ${ }^{1}$ Silvia Forni, ${ }^{2}$ Alessandro Morettini ${ }^{1}$ \\ ${ }^{1}$ Dipartimento di Medicina Interna, AOU Careggi, Firenze; ${ }^{2}$ Agenzia Regionale di Sanità Toscana, Firenze, Italy
}

\begin{abstract}
Rehospitalization is the return of a patient to a surgical or medical department within 30 days from discharge. We have limited information on the frequency and patterns of rehospitalization in Italy. Our purpose is to describe this phenomenon in Tuscany. We analyzed regional hospital discharge abstract data in Tuscany 2012, to describe 30-day all cause readmissions. We studied patients aged 18 or more $(11,846)$ discharged with diagnoses of heart failure and pneumonia from Internal Medicine Departments. Relationships between 30-day readmission rates, demographic and clinical characteristics have been analyzed. $18 \%$ and $15 \%$ of subjects respectively discharged with diagnosis of heart failure and pneumonia were readmitted within 30 days. Risk factors significantly related to readmissions were longer length of stay, increasing number of different medications taken during the year preceding hospitalization and greater number of hospitalizations during the semester preceding admission. From the analysis of risk factors we found that frailty and complexity of patients (identified by long hospitalization stays, high number of drugs and previous admissions) are the most important factors for unplanned readmissions.
\end{abstract}

\section{Introduction}

Rehospitalization is the return of a patient to a surgical or medical department within 30 days from discharge.

Reducing rates of rehospitalization have attracted attention as a way to improve quality of care and reduce costs in the USA. We have limited information on the

Correspondence: Matteo Tellini, p.zza Kennedy 14, 50018 Scandicci (FI), Italy.

E-mail: mattetello@gmail.com

Key words: Rehospitalization; 30-day readmission rates; revolving door syndrome; internal medicine.

Acknowledgments: the authors thank the Agenzia Regionale di Sanità Toscana and Dr. Andrea Vannucci for the help in data collection and statistical analysis.

Conflict of interest: the authors declare no potential conflict of interest.

Received for publication: 24 February 2014.

Revision received: 8 April 2014.

Accepted for publication: 21 April 2014.

This work is licensed under a Creative Commons Attribution NonCommercial 3.0 License (CC BY-NC 3.0).

CCopyright M. Tellini et al., 2015

Licensee PAGEPress, Italy

Italian Journal of Medicine 2015; 9:150-156

doi:10.4081/itjm.2015.488 frequency and patterns of rehospitalization in Italy.

Our purpose is to describe the situation of readmissions in Tuscany especially in the population of patients discharged from Internal Medicine wards.

\section{Materials and Methods}

We used data from the regional hospital discharge abstract (HDA) of Tuscany, Italy, between 2010-2012. The index admissions included patients aged 18 years or more, admitted in any Internal Medicine unit of Tuscany with principal diagnosis of heart failure or pneumonia and discharged alive from $1^{\text {st January }} 2012$ to $30^{\text {th }}$ November 2012. Exclusion criteria were staying outside Tuscany, transfer to another acute care hospital, death during hospitalization and discharge against medical advice. Eligible index admissions within 30 days from an index discharge were also excluded. Readmission was defined as an admission for any cause within 30 days from the data of index discharge. Readmissions in hospitals outside Tuscany were included. If patients had two or more readmissions within 30 days of discharge from the index admission only one was counted as a rehospitalization; every readmission beyond 30 days from index discharge was counted as a new index hospitalization. We also included planned readmissions, although they were not numerous (i.e., placement of pacemaker after hospitalization for heart failure).

Data about comorbidities, medical or surgical pro- 
cedures, prior hospitalizations and drugs assumption for each patient were obtained from HDA and drug prescription abstract in the two previous years. Demographic and clinical characteristics, time to, diagnosis and place of readmission were also analyzed.

We also decided to adjust readmission rates for age, removing its possible effect on rehospitalization risk; so we stratified population of subjects discharged with diagnoses of heart failure and pneumonia into several age classes and we calculated relative readmission risk of every class in respect to the youngest group ( $<45$ years).

Univariate and multivariate odds ratios have been computed to estimate association between risk factor and readmissions. Data were analyzed using STATA-SE (version 12.1, StataCorp LP, College Station, TX, USA).

\section{Results}

\section{Thirty-day readmission rates in patients discharged with diagnosis of heart failure}

In 2012 we accounted 7056 patients discharged with diagnosis of heart failure from the totality of Internal Medicine Departments of Tuscany (3008 males and 4048 females). Mean age was 82.1 years [standard deviation (SD) \pm 8.7 years]. Thirty-days readmission rate was $18.3 \%$.

The 30 -day mortality rate was $12.8 \%$ and it has been calculated as the ratio between patients died within 30 days from admission and the totality of patients discharged with diagnosis of heart failure.

An increasing number of hospitalizations suffered during the 6 months before index hospitalization and a larger number of different drugs taken in the year preceding index discharge are statistically related to rehospitalization risk, in both the univariate and multivariate analyses we conducted (Table 1).

Age does not represent a significant factor in determining rehospitalization as we can observe in the final multivariate model.
During the univariate analysis (Table 1) we found that readmission risk significantly increases with longer lengths of stay. Mean length of stay for patients who were readmitted was 8.4 days versus 7.5 days for those who were not readmitted.

We think that this result means that patients who present longer lengths of stay have acute diseases that require more days to stabilize the clinical status. Therefore these patients are more frail and have an increased risk of readmission.

Although we use to think that many patients come back to hospital within very few days from discharge because of unstable clinical status, our results show inverse tendency: most of rehospitalization occur between 8 and 30 days from discharge.

Probably rehospitalizations are more related to illness severity than to inadequate in-hospital management.

We observed that heart failure was the most important cause of readmission in patients discharged with diagnosis of heart failure (Table 2), accounting for more than one third of the readmissions, as described in other studies. ${ }^{1}$

With the intent to establish a risk profile of patient undergoing a rehospitalization, we studied the relationship between readmission risk and a large number of comorbidities and in-hospital procedures.

Multivariate analysis shows that cardiovascular diseases (previous cardiac and great vessels surgery, valvulopathies, placement of pacemaker or implantable cardioverter defibrillator, atherosclerosis, vasculitis, aortic aneurysm, aortic dissection, etc.) and hematologic abnormalities (anemia, aplastic anemia, white cells diseases except leukemia) are significantly related to unplanned readmissions risk.

We stratified population into age classes, as presented in Table 3, and estimated relative risk of rehospitalization between class of aged 19-45 and elder groups, so that we removed age effect as responsible of excess of hospitalization.

After adjusting for age, comorbidities and factors which continue to affect readmission risk are chronic kidney disease (except in the youngest and eldest age

Table 1. Relationship between 30-day readmission rate and continue variables in subjects discharged with diagnosis of heart failure.

\begin{tabular}{|c|c|c|c|c|c|c|}
\hline \multirow{2}{*}{$\overline{\text { Gender }}$} & \multirow{2}{*}{$\begin{array}{c}30 \text { days } \\
\text { readmissions (\%) }\end{array}$} & \multirow{2}{*}{$\begin{array}{c}\text { Total of } \\
\text { discharges }(\%)\end{array}$} & \multirow[t]{2}{*}{ OR } & \multicolumn{2}{|c|}{$95 \% \mathrm{CI}$} & \multirow[t]{2}{*}{ P-value } \\
\hline & & & & & & \\
\hline Male & $606(20)$ & 3008 (43) & - & - & - & 0.001 \\
\hline Female & $685(17)$ & $4048(57)$ & 0.807 & 0.715 & 0.911 & - \\
\hline Length of stay & $1291(18)$ & $7056(100)$ & 1.030 & 1019 & 1.041 & 0.000 \\
\hline No. of different drugs taken in the past year & $1265(18)$ & $6859(100)$ & 1.034 & 1.025 & 1.043 & 0.000 \\
\hline Previous hospitalization & $1291(18)$ & $7056(100)$ & 1.127 & 1.105 & 1.149 & 0.000 \\
\hline
\end{tabular}

OR, odds ratio; CI, confidence interval. 
groups) and continue variables like increasing length of stay, number of hospitalizations in the semester preceding index hospitalization and number of different active principles taken during the year preceding index discharge.

\section{Thirty-day readmission rates in patients discharged with diagnosis of pneumonia}

Patients discharged from every Internal Medicine Department of Tuscany with diagnosis of pneumonia in 2012 were 4790, of which 2542 males $(53 \%)$ and 2248 females (47\%). Mean age was 78.0 years ( $\mathrm{SD} \pm 13.1$ years).

A number of 726 patients were readmitted within 30 days from discharge, so that rehospitalization rate for this population was $15.2 \%$.

The 30-day mortality rate from hospitalization (calculated as presented in the paragraph concerning heart failure) was $14.5 \%$.

Age does not affect readmission risk for patients discharged with diagnosis of pneumonia and although it is a significant factor at univariate analysis, its importance vanishes at multivariate analysis (Table 4).

An increasing number of different drugs assumed and a larger number of hospitalizations suffered in the months preceding index hospitalization are strictly related to 30-day readmission risk. This finding strengthens our conviction that frail patients, affected by several diseases and medicated with multiple different drugs, are at high risk of being rehospitalized.

As longer length of stay, as greater readmission risk: a long length of stay could indicate a quite serious underlying disease, which could be accountable for an exceeding number of hospitalizations.

Mean length of stay for patients discharged with diagnosis of pneumonia and later readmitted in hospital is longer than the length of stay for patients who do not suffer a rehospitalization (10.6 days vs 9.0 days).

Patients discharged with diagnosis of pneumonia from any Internal Medicine Department of Tuscany are more frequently readmitted within 8 and 30 days from discharge, according to our findings in heart failure population.

The most frequent rehospitalization diagnosis of population studied is pneumonia: patients are more often rehospitalized for the same reason of index discharge (Table 4).

Multivariate analysis shows that neoplasia, diabetes mellitus and hematologic abnormalities (anemia, aplastic anemia, white cells diseases except leukemia) are significantly related to unplanned readmissions risk.

Some of the studied procedures have been statistically related to readmission risk, in particular percutaneous transluminal coronary angioplasty (PTCA) interventions and central venous line placement.

We can think comorbidities to exert a greater in- fluence on readmission risk for patients discharged with diagnosis of pneumonia than for those discharged with diagnosis of heart failure. Pneumonia, an acute and potentially resolvable disease, unlike heart failure, should not bring itself to higher readmission rates: comorbidities instead, expressing clinical patient complexity, could more seriously affect those rates.

We stratified population into age classes and estimated relative risk of rehospitalization between class of aged 19-45 and elder groups. So that we removed age effect as responsible for excess of hospitalization.

After adjusting for age, comorbidities like neoplasia, diabetes and hematological diseases continue to

Table 2. Most important causes of readmission in subjects discharged with diagnosis of heart failure.

\begin{tabular}{lcc}
\hline Diagnosis of readmission & Frequency & $\mathbf{\%}$ \\
\hline Heart failure & 440 & 34.58 \\
\hline Acute respiratory failure & 114 & 13.18 \\
\hline Pneumonia & 50 & 5.78 \\
\hline Acute respiratory failure in COPD & 29 & 3.35 \\
\hline Acute kidney injury & 29 & 3.35 \\
\hline Aortic valve disease & 20 & 2.31 \\
\hline Decompensated COPD & 14 & 1.62 \\
\hline Acute pulmonary edema & 14 & 1.62 \\
\hline Myocardial infarction & 13 & 1.50 \\
\hline Atrial fibrillation & 13 & 1.50 \\
\hline Acute coronary syndrome & 11 & 1.27 \\
\hline Stroke & 11 & 1.27 \\
\hline Pulmonary embolism & 10 & 1.16 \\
\hline Mitral valve disease & 10 & 1.16 \\
\hline Clostridium difficile infection & 9 & 1.04 \\
\hline Sepsis & 9 & 1.04 \\
\hline Dehydration & 9 & 1.04 \\
\hline CorD chic obs & & \\
\hline
\end{tabular}

COPD, chronic obstructive pulmonary disease.

Table 3. Age classes of population of subjects discharged with diagnosis of heart failure and 30-day mortality rates.

\begin{tabular}{lcc}
\hline Age & $\begin{array}{c}\text { 30-day readmission } \\
\text { rates }\end{array}$ & $\begin{array}{c}\text { 30-day mortality } \\
\text { rates }\end{array}$ \\
\hline$<45$ years & $16 \%$ & $0 \%$ \\
\hline $45-64$ years & $21 \%$ & $2 \%$ \\
\hline $65-74$ years & $21 \%$ & $4 \%$ \\
\hline $75-84$ years & $19 \%$ & $6 \%$ \\
\hline$\geq 85$ years & $17 \%$ & $9 \%$ \\
\hline
\end{tabular}


affect readmission risk. So that we could deduce that increasing readmission risk for elder groups is not due to age factor, but to comorbidity effect.

In addition continue variables like increasing length of stay, number of hospitalizations in the semester preceding index hospitalization and number of different active principles taken during the year preceding index discharge continue to affect readmission risk.

In conclusion, factors significantly linked to readmission risk are listed in Table 5 that presents results of multivariate analysis.

C-statistic of our model was 0.65 therefore not a high discriminative capability, but near to results of other important studies dealing with the problem of rehospitalization.

\section{Discussion}

Our study tried to describe the problem of rehospitalization in Internal Medicine Departments of our region, in particular for subjects discharged with diagnosis of heart failure and pneumonia.

Age, usually conceived as one of the most important factor for readmissions, is not significantly related to this phenomenon at multivariate analysis and its importance gives way to other factors. This result complies with the findings of other authors. ${ }^{1-5}$

As described by Jencks et al., ${ }^{1}$ at our analysis male sex is a statistically significant risk factor for unplanned readmissions in subjects discharged with diagnosis of heart failure. Prognosis of cardiovascular diseases is usually better for females ${ }^{6,7}$ and differences in outcomes such as mortality or rehospitalization are probably attributable to differences in etiology of heart failure (arterial hypertension in females versus coronary artery disease in male subjects). ${ }^{8}$

We supposed sex differences related to differences in compliance with chronic therapies: furthermore any study ${ }^{9-11}$ reported significant variance between sex and adherence to medications. Rather lack of compliance is statistically associated with major complexity of therapeutic plans.

Number of hospitalizations and number of different drugs assumed respectively in the semester and in the year preceding index discharge were statistically related to readmission risk in both populations we studied at multivariate analysis. These factors could represent markers of complexity and frailty of patients and they may account for the total burden of illness, illness

Table 4. Most important causes of readmission in subjects discharged with diagnosis of pneumonia.

\begin{tabular}{lcc}
\hline Diagnosis of readmission & Frequency & \% \\
\hline Pneumonia & 139 & 19.42 \\
\hline Acute lung injury & 77 & 12.94 \\
\hline Heart failure & 48 & 8.07 \\
\hline Acute on chronic respiratory failure & 24 & 4.03 \\
\hline Clostridium difficile infection & 12 & 2.02 \\
\hline Sepsis & 12 & 2.02 \\
\hline Acute kidney injury & 11 & 1.85 \\
\hline Exacerbation of COPD & 10 & 1.68 \\
\hline Dehydration & 9 & 1.51 \\
\hline Pleural effusion & 9 & 1.51 \\
\hline Pulmonary embolism & 6 & 1.01 \\
\hline Dysphagia & 6 & 1.01 \\
\hline Intestinal obstruction & 6 & 1.01 \\
\hline Acute leukemia & 5 & 0.84 \\
\hline Alzheimer disease & 5 & 0.84 \\
\hline Intracranial atherosclerosis & 5 & 0.84 \\
\hline
\end{tabular}

COPD, chronic obstructive pulmonary disease.

Table 5. Predictors of unplanned 30-day readmissions in subjects discharged with diagnosis of pneumonia (multivariate analysis).

\begin{tabular}{|c|c|c|c|c|}
\hline \multirow[b]{2}{*}{ Neoplasia } & \multirow{2}{*}{$\begin{array}{c}\text { OR } \\
1.369\end{array}$} & \multirow{2}{*}{$\begin{array}{c}\mathbf{P}>|\mathbf{z}| \\
0.015\end{array}$} & \multicolumn{2}{|c|}{$95 \%$ CI } \\
\hline & & & 1.063 & 1.765 \\
\hline Diabetes & 1.168 & 0.146 & 0.948 & 1.440 \\
\hline Hematological disease & 1.358 & 0.006 & 1.091 & 1.691 \\
\hline Previous PTCA & 1.545 & 0.190 & 0.806 & 2.959 \\
\hline Venous central line placement during hospitalization & 1.635 & 0.010 & 1.125 & 2.374 \\
\hline Length of stay & 1.029 & 0.000 & 1.017 & 1.040 \\
\hline No. of different drugs taken & 1.017 & 0.006 & 1.005 & 1.030 \\
\hline No. of hospitalizations & 1.176 & 0.000 & 1.127 & 1.228 \\
\hline
\end{tabular}

OR, odds ratio; CI, confidence interval; PTCA, percutaneous transluminal coronary angioplasty. 
severity, functional status and/or social environment. ${ }^{5}$

Length of stay is an important factor influencing return to hospital: every day of hospitalization brings to an increase of rehospitalization risk. So it is not true that patients discharged too early come back to hospital more frequently than patients held in hospital more days, as we thought at the beginning: length of stay reflects severity of clinical status and, therefore, an increased rehospitalization risk. Importance of this factor on readmission risk is also recognized in many studies in literature..$^{1,3,5}$

In most of cases readmission and discharge diagnoses coincide, as we can find in the work of Jencks et al. ${ }^{1}$

As expected on the basis of literature review, few comorbidities were significantly related to readmission risk, such as cardiovascular and hematologic diseases for subjects discharged with diagnosis of heart failure; neoplasia, diabetes mellitus and hematologic abnormalities for those discharged with diagnosis of pneumonia.

This result confirms difficulty in finding patients at risk of readmission on the basis of clinical data. It is possible, as presented in several studies, that length of stay or number of previous hospitalizations could reflect the severity and the instability of patients better than comorbidities ${ }^{5}$ or primary discharge diagnosis ${ }^{2}$ do.

Some authors ${ }^{12}$ underlined the importance of considering that patients hospitalized in Internal Medicine wards are more and more older. In elderly, in addition to comorbidities, many age-related impairments complicate patients' clinical and functional status. Complexity of patients is not exclusively defined by comorbidities (although they might contribute to readmissions) but also by social and family support, severity of illness, degree of stability, cognitive and functional status, compliance to therapy, mobility, feeding ability, presence of bed sores, sphincter incontinence, etc. A multidimensional and interdisciplinary approach is mandatory in order to guarantee a high quality health care. The efforts of internists should go beyond diagnosis and therapy for acute events and they should aim to optimize and preserve functional status.

Only few procedures we studied were statistically significant for readmission risk: neither PTCA nor coronary artery bypass graft were related to readmission risk in patients discharged with diagnosis of heart failure. For subjects with diagnosis of pneumonia at discharge placement of central venous lines or coronary revascularization (PTCA) were associated with rehospitalization risk at multivariate analysis. Unexpectedly $\mathrm{O}_{2}$-therapy, non-invasive ventilation (NIV) or intravenous antibiotic infusion did not achieve statistical significance: although not logical on clinical basis, this finding could reflect a lacking HDA compiling at the moment of discharge or a not yet widespread use of NIV in Internal Medicine Departments of Tuscany. Some studies 5 included the number of procedures performed during the index stay among risk factors for avoidable readmissions: depending on the procedure, this predictor may reflect disease severity.

Given the complexity of identifying a risk profile of patient undergoing a rehospitalization, we would like to concentrate on a common moment during hospitalization: the moment of discharge. Several international studies suggest it could be very precious to pay attention to this process which too often is put in hands of subjectivity and good sense of the doctor who is going to discharge the patient.

The re-engineered discharge (RED) project, conducted by Boston University, ${ }^{13}$ analyzed the effects of the current discharge program in USA: from 19 to $23 \%$ of patients will face an adverse event post-discharge. Potential critical points of discharge process are identified in: physician-patient communication, documentation released at the moment of discharge, reconciliation of therapy, pending results of diagnostic exams performed during hospitalization, planning follow-up post-discharge, training of patient outgoing to extra-hospital setting. For these reasons the RED project authors edited a checklist with the intent to make the discharge program more standardized and objective: i) patient education throughout hospital course; ii) schedule follow-up appointments - physician visits and tests; iii) follow up on outstanding test results; iv) organize post-discharge services; v) confirm medication plan - reconcile discharge medications; vi) reconcile discharge plan with national guidelines; vii) review steps for what to do if a problem arises; viii) transmission of discharge summary to primary care physician; ix) assess patient understanding of discharge plan; $x$ ) give written discharge plan; xi) provide telephone reinforcement.

The RED project, published in January 2013, was tested into a randomized controlled trial at the Boston Medical Center, involving 750 subjects: half of the sample was exposed to conventional discharge program, while other half underwent the reviewed and standardized program. Results of the trial were very satisfying: number of visits in Emergency Unit and number of rehospitalization significantly decreased from $166(45.1 \%)$ to 145 (31.4\%) (P-value $<0.009)$, saving up to 400 US dollars for each patient discharged using RED procedure.

A recent review ${ }^{14}$ showed that case management did not reduce emergency admissions or unplanned readmissions in people aged $\geq 65$ years. Case management was considered as a collaborative process in the care of patients that includes planning, coordinating and reviewing care. The meta-analysis did not include RED project - just discussed above - which emphasizes patient and caregiver education and coaching, in contrast with traditional approach of case management 
which focuses on matching community resources to patient needs. Engagement of patients themselves in management of their chronic diseases could be a key factor contributing to reduce hospital utilization and improve quality of care.

However several studies ${ }^{15,16}$ suggest that using readmission rates as a measure of quality of care and as a criteria for hospital reimbursement/taxation (as in the USA) would not be appropriated. A similar use of these rates presumes that a large number of readmissions are avoidable by improving management in and outside hospitals. Unfortunately, as reported by our study and by other important studies in literature, factors influencing a rehospitalization are various and numerous (correlated to clinical or demographical aspects and to patterns of utilization of health systems) and not completely controllable.

Several studies tried to identify patients at risk of avoidable readmissions using simple scores (based on clinical and administrative data easily available during hospitalization or at the time of discharge) in order to address resource-intensive transitional care interventions focusing on high-risk patients. Donzé et al., for example, reported that avoidable readmissions were $36.7 \%$ of all readmissions, ${ }^{5}$ in keeping with average rate presented in literature ${ }^{17}(27.1 \%)$. Process of labelling a readmission as avoidable is furthermore subjective and conditioned to the opinion of reviewer.

Because only $27.1 \%$ of readmission is deemed avoidable ${ }^{17}$ and constant among different centers (whereas rehospitalization rates are extremely various in different sites), some authors ${ }^{18}$ asked if readmission rates are a good tool for benchmarking across hospitals. Factors causing rehospitalization are often out of hospital's control (i.e., mental illness, poor social support and poverty). Are high readmission rates always expression of poor health care quality? Some centers might be able to keep healthiest people far from hospitals or could show lower mortality rates or better access to hospital care and, conversely, they could present a higher number of rehospitalization. From this viewpoint, many authors underline the importance of not focusing only on phenomenon of readmissions and they suggest to improve quality of care by paying attention to other problems (sometimes more preventable than readmissions) such as patient safety during hospitalizations.

Readmission risk persists beyond time window studied and improvement of quality of care should concern not only the first 30 days after discharge for subjects affected by chronic diseases.

\section{Conclusions}

Our study allowed to describe phenomenon of rehospitalization in Italy, in which it was poorly studied and known until now.
Numerousness of studied population makes our data interesting and statistically reliable.

As reported by other important studies in literature, we found that it is really hard to draw an identikit profile of patients at risk of readmission on the basis of demographic, social or clinical data.

The most important factors influencing the excess of hospitalizations (length of stay, number of different drugs, number of previous hospitalizations) represent, in our opinion, the frailty and the complexity of patients.

This study could be the basis for further investigations in order to know if improving discharge program could reduce readmission rates.

\section{References}

1. Jencks SF, Williams MV, Coleman EA. Rehospitalizations among patients in the Medicare fee-for-service program. N Engl J Med 2009;360:1418-28.

2. Bradley EH, Yakusheva O, Horwitz LI, et al. Identifying patients at increased risk for unplanned readmission. Med Care 2013;51:761-6.

3. Hasan O, Meltzer DO, Shaykevich SA, et al. Hospital readmission in general medicine patients: a prediction model. J Gen Intern Med 2010;25:211-9.

4. Philbin EF, Di Salvo TG. Prediction of hospital readmission for heart failure: development of a simple risk score based on administrative data. J Am Coll Cardiol 1999;33:1560-6.

5. Donzé J, Aujesky D, Williams D, et al. Potentially avoidable 30-day hospital readmissions in medical patients: derivation and validation of a prediction model. JAMA Intern Med 2013;173:632-8.

6. Ho KK, Anderson KM, Kannel WB, et al. Survival after the onset of congestive heart failure in Framingham Heart Study subjects. Circulation 1993;88:107-15.

7. McKee PA, Castelli WP, McNamara PM, et al. The natural history of congestive heart failure: the Framingham study. N Engl J Med 1971;285:1441-6.

8. Adams KF Jr, Sueta CA, Gheorghiade M, et al. Gender differences in survival in advanced heart failure. Insights from the FIRST study. Circulation 1999;99:1816-21.

9. Balkrishnan R. Predictors of medication adherence in the elderly. Clin Ther 1998;20:764-71.

10. Coons SJ, Sheahan SL, Martin SS, et al. Predictors of medication noncompliance in a sample of older adults. Clin Ther 1994;16:110-7.

11. Osterberg L, Blaschke T. Adherence to medication. N Engl J Med 2005;353:487-97.

12. Nardi R, Scanelli G, Borioni D, et al. The assessment of complexity in internal medicine patients. The FADOI Medicomplex Study. Eur J Intern Med 2007; 18:283-7.

13. Manasseh C. Implementing an evidence based hospital discharge process. Learning from the experience of project re-engineered discharge (RED); 2013. Available from: http://www.omh.ny.gov/omhweb/psyckes_medicaid/initiatives/hospital/learning_collaborative_2013/call s/BostonUniversity.pdf

14. Huntley AL, Thomas R, Mann M, et al. Is case manage- 
ment effective in reducing the risk of unplanned hospital admissions for older people? A systematic review and meta-analysis. Fam Pract 2013;30:266-75.

15. Clarke A. Are readmissions avoidable? BMJ 1990;301: 1136-8.

16. Goodman DC, Fisher ES, Chang CH. The revolving door: a report on U.S. hospital readmissions. Dartmouth
Atlas Project; 2013. Available from: http:/www.rwjf. org/content/dam/farm/reports/reports/2013/rwjf404178

17. van Walraven $C$, Bennett $C$, Jennings A, et al. Proportion of hospital readmissions deemed avoidable: a systematic review. CMAJ 2011;183:E391-402.

18. Joynt KE, Jha AK. Thirty-day readmissions - truth and consequences. NEJM 2012;366:1366-9. 